\title{
Human antiaging research: a viewpoint from food science on calorie restriction mimetics
}

\author{
${ }^{1,2}$ Shintani, T. \\ ${ }^{1}$ Department of Nutritional Representative - Supplement Adviser, The Japanese Clinical Nutrition \\ Association, Tokyo 153-0044, Japan \\ ${ }^{2}$ Graduate School of Agricultural Science, Ehime University, Matsuyama 790-8577, Japan
}

\author{
Article history: \\ Received: 24 May 2020 \\ Received in revised form: 1 \\ July 2020 \\ Accepted: 16 July 2020 \\ Available Online: 2 \\ September 2020 \\ Keywords: \\ Calorie restriction, \\ Antiaging, \\ Calorie restriction mimetics, \\ Glucose, \\ Dietary supplements, \\ Food research
}

DOI:

https://doi.org/10.26656/fr.2017.4(6).250

\begin{abstract}
Calorie restriction (CR) is to reduce food intake without malnutrition and is sometimes adopted intentionally to reduce body weight for improving health. CR has been known to prolong the lifespan in several animal models such as nematodes, flies, and mice. While it is not feasible to implement long term CR in humans, the intake of specific food substances that produce the same effect as CR can result in significant health benefits to humans. This paper highlights the need for promoting research on such calorie restriction mimetics (CRMs). This scientific opinion suggests that the promotion of research on CRMs derived from foods and food resources has the potential to enhance health and prolong life span. Collaboration between researchers from the fields of food science and those working on CRMs has the potential to promote good health and enhance human longevity in the aging society.
\end{abstract}

\section{Introduction}

Why do we explore the function of food? The function of food is explored not only to elucidate the efficacy of foods but also to promote health through conducting basic food research that would lead to a healthily aging community. In aging research, only one concept on the dietary regimen for longevity mentioned below has gained remarkable consensus. The most trusted method for healthy aging is the dietary restriction or calorie restriction (CR) (Shintani et al., 2018; Madeo et al., 2019). CR is a dietary regimen that reduces food intake without incurring malnutrition. $\mathrm{CR}$ is sometimes adopted intentionally to control body weight so that it could improve health. CR was shown to prolong the lifespan of experimental animals, such as nematodes, flies, and mice (Shintani et al., 2018). In a substantial first clinical study, CR was reported to delay the progression of diverse age-related changes (Martin et al., 2016). Even if CR prolongs human lifespan, it is difficult to conduct long-term CR experiments in humans. Therefore, it is preferable to develop a method or compound that reproduces the effect of $\mathrm{CR}$ without limiting dietary intake.

\section{Research on calorie restriction mimetics}

The concept of CR mimetics (CRMs) was proposed by Lane et al. (1998). Recent research has shown that CRMs will eventually be considered among the group of substances effective against age-related diseases, such as cardiovascular and neurodegenerative diseases (Martin et al., 2016). In addition to major CRMs, such as 2-DG (2deoxy glucose) (Lane et al., 1998) that modulates glucose metabolism, CRMs include compounds affecting nutrient sensing signals and histone acetylation; due to these compounds, the concept of CRM is becoming popular. CRMs include resveratrol, spermidine, oxaloacetic acid, and D-glucosamine (Figure 1), which

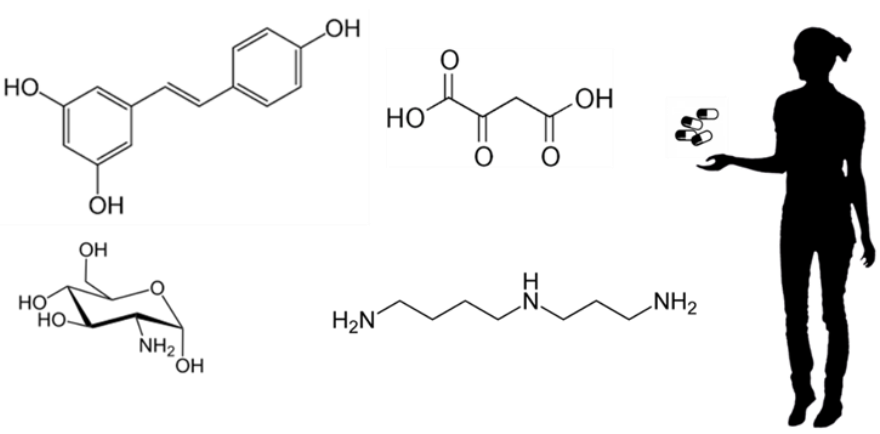

Figure 1. CRMs, dietary supplements, and human. Molecular structures of several CRMs are shown. Left-upper, resveratrol; right-upper, oxaloacetic acid; left-lower; D-glucosamine; rightlower, spermidine. 
are food components showing antiaging and longevitypromoting effects (Shintani, Kosuge and Ashida, 2018; Madeo et al., 2019).

There are several compounds that can act as CRMs exhibiting functions different from those of the compounds mentioned above. Some CRMs are made from petrochemicals, the social acceptance of which seems low; hence, they may not be practical for human use. However, food-derived components will most likely be readily applicable.

Unfortunately, many studies of food functionality were oriented towards healthy longevity without mentioning the concept of CRM. Thus food-derived CRMs studies and food science studies have conducted separately over the years. Researchers from the areas of food science should further contribute to the field of CRMs, as understanding the food-derived CRMs well is very important for increasing their application. For example, food substances that do not undergo changes or oxidation easily under normal temperature, normal pressure, and low oxygen concentration, should be researched. Such substances will have a low final cost owing to their stable properties. Furthermore, these potential CRMs should be easy to access and produce, so that they can be made readily available in the market. The methods for the production of substances, such as functional hexoses (e.g., D-glucosamine), are being researched and developed (Shintani, 2019); however, further research on production methods is still required for many other compounds. Thus, although the function of CRMs is established, their practical application will not be possible until the chemical properties of the CRMs are understood and they are easily produced at low cost. Furthermore, applied research on processed food containing CRMs and on CRMs alone is also extremely important for attaining health and longevity in the society.

Although excessive dietary intake has been reported to impair health and cause obesity and diabetes worldwide, it is difficult to reduce daily dietary intake immediately and prevent lifestyle-related diseases. It is relatively easier for most people to consume additional foods, dietary supplements, or medicines derived from foods. When researchers are working with health food ingredients from the viewpoint of food science, they should check whether the ingredients are on the CRM list (Shintani et al., 2018; Madeo et al., 2019). If this is not the case, collaboration with antiaging researchers working with CRMs can promote social awareness of their research, and in turn, help achieve the goal of a society with enhanced longevity. When publishing a paper on food ingredients, researchers should mention that the item being listed is a CRM, if it meets the criteria. This can connect their conventional food research with CRM research, leading to greater success in terms of increased human longevity.

\section{Conclusion}

The aim of this scientific opinion is to indicate that promotion of research on CRMs derived from foods and food resources has the potential to enhance health and prolong life span; the expansion of the applications of CRMs in the food industry would help promote health. Besides research on the functionality of CRMs oriented towards healthy longevity, studies on such as their chemical properties and production methods are necessary for practical use of food-derived CRMs in the society.

\section{Conflict of interest}

The author declares no competing financial interest. Tomoya Shintani is an employee of Matsutani Chemical Industry Co., Ltd. (Hyogo, Japan). However, the company provided no financial support for this short communication.

\section{Acknowledgments}

The author thanks Hisashi Ashida (Kindai University, Wakayama, Japan), Masashi Sato (Kagawa University, Kagawa, Japan), and Hideya Shintani (Osaka Saiseikai Izuo Hospital, Osaka, Japan) for valuable advice. This study was partially supported by the Sasakawa Scientific Research Grant from The Japan Science Society [2018-6021].

\section{References}

Lane, M.A., Ingram, D.K. and Roth, G.S. (1998). 2Deoxy-D-glucose feeding in rats mimics physiologic effects of calorie restriction. Journal of Anti-Aging Medicine, 1(4), 327-337. https://doi.org/10.1089/ rej.1.1998.1.327

Madeo, F., Carmona-Gutierrez, D., Hofer, S.J. and Kroemer, G. (2019). Caloric restriction mimetics against age-associated disease: targets, mechanisms, and therapeutic potential. Cell Metabolism, 29(3), 592-610. https://doi.org/10.1016/j.cmet.2019.01.018

Martin, C. K., Bhapkar, M., Pittas, A. G., Pieper, C. F., Das, S. K., Williamson, D. A., Scott, T., Redman, L. M., Stein, R., Gilhooly, C. H., Stewart, T., Robinson, L., Roberts, S. B. and Mayer, J. (2016). Effect of calorie restriction on mood, quality of life, sleep, and sexual function in healthy nonobese adults: The CALERIE 2 randomized clinical trial for the 
comprehensive assessment of long-term effects of reducing intake of energy (CALERIE) phase 2 study group. JAMA Internal Medicine, 176(6), 743-752. https://doi.org/10.1001/jamainternmed.2016.1189

Shintani, H., Shintani, T., Ashida, H. and Sato, M. (2018). Calorie restriction mimetics: upstream-type compounds for modulating glucose metabolism. Nutrients, 10(12), 1821. https://doi.org/10.3390/ nu10121821

Shintani, T., Kosuge, Y. and Ashida, H. (2018). Glucosamine Extends the Lifespan of Caenorhabditis elegans via Autophagy Induction. Journal of Applied Glycoscience, 65(3), 37-43. https://doi.org/10.5458/ jag.jag.JAG-2018_002

Shintani, T. (2019). Food industrial production of monosaccharides using microbial, enzymatic, and chemical methods. Fermentation, 5(2), 47. https:// doi.org/10.3390/fermentation5020047 